\title{
Primary screening method for predicting onset of eating disturbances in female high school students
}

\author{
Kazue HIROKANE and Mikako ARAKIDA
}

The purpose of this study is to find out an better screening protocol to identify female adolescents at risk for eating disturbances.

We administered a newly-developed eating disturbance onset prediction test and the Eating Attitude Test-26（the EAT-26） to 1061 Japanese female high school students at Time 1 (T1). EAT-26 was re-administered about 10 months later, designated as Time 2 (T2). Based on EAT-26 findings, students were classified into four eating behavior groups : onset-free group, recovered group, persistent group, and onset group. The normal groups at T1 (onset-free and onset groups) were extracted in Step 1, and the onset group was predicted from the extracted normal groups in Step 2. Useful items for Step 2 were investigated through the eating disturbance onset prediction test, and four items were selected. As a result, the correct discrimination rate in the onset group was obtained as $73.3 \%$, and the negative predictive value was obtained as $98.3 \%$.

These results show that the protocol using the four items is useful in predicting the onset of eating disturbances among female high school students. False negative cases accounted for $1.2 \%$ of all students, thus, reduction of the false-negative rate by modification of measurement items may be needed.

Key words : eating disorders, eating disturbances, screening test, adolescents girls, the EAT-26

\section{Introduction}

Eating disorders (ED) frequently occur in females in adolescence and young adulthood, and their rapid increase in recent years has been reported (Kiriike, 2005 ; Nakai, 2000). Once ED occur, remission and recurrence repeat, and the disease type becomes complex along with the course. Symptoms are likely to become fixed, and treatment is difficult in many cases. Thus, it is important to take preventive measures for $\mathrm{ED}$, in addition to early detection.

In Japan, an early detection protocol for ED by physical measurements values and interviews in a school care setting has been developed, which has provided good results (Tanaka et al., 2004 ; Watanabe and Tokumura, 2005). Regarding preventive measures, the practice of preventive education (Nagai et al., 2005) has been reported, but is currently limited to "universal prevention programs," in which general contents are applied to the whole population regardless of the presence or absence of symptoms and the degree of risk.

ED became a social problem 20-30 years earlier in Europe and America than in Japan, and preventive education and its long-term evaluation have been performed. "Universal prevention programs" and "selected prevention programs" consisting of

Osaka University Graduate School of Medicine, Division of Health Sciences 
contents corresponding to extracted high-risk groups were compared, and it was concluded that "selected prevention programs" are more effective as preventive education (Stice and Shaw, 2004). "Selected eating disorder prevention programs" have not been performed in Japan, one reason for this is a lack of established screening methods for Japanese high-risk eating disorder groups.

As the number of patients increased, ED has been clarified to develop various symptoms in which the pathological condition changes with the course of morbidity. The American Psychiatric Association has continuously revised the diagnostic criteria since it first published the concept of $\mathrm{ED}$ in the "Diagnostic and Statistical Manual of Mental Disorders" in 1980. Cases that did not meet the criteria of ED also emerge mental and physical consequences similar to those of ED (Crow et al., 2002 ; Johnson et al., 2002), and a higher incidence of such cases than those of typical anorexia nervosa and bulimia nervosa has been reported (Kiriike, 2005). Moreover, of adolescent girls recommended to be treated at eating disorder-specialized clinics, those not meeting the criteria accounted for substantial proportion (Bunnell and Shenker, 1990 ; Mitrany, 1992).

To date, there is no consensus as to the definitions of such partial ED, but it has been reported that individuals with partial ED are at risk of progressing to full-syndrome ED from the viewpoint that normal eating is continuous to full-syndrome ED (Weber et al., 2005). In this study, we focused on such partial ED with severe symptoms almost similar to those of ED, designated as "eating disturbances," as the screening target.

The goal of the study was to develop a protocol for predicting the onset of eating disturbances in adolescence and to investigate its usefulness. This study is significant because it contributes to the primary prevention of ED by extracting the high- risk group that may develop eating disturbances, thus enabling delivery of onset-preventive programs for the high-risk group.

\section{Methods}

\section{Subjects}

The subjects were 1278 first-year female high school students attending six public and one private school in the Kanto and Kansai areas in Japan.

\section{Measures}

1) Eating behavior rating scale

For the evaluation of eating attitudes and behaviors, the Eating Attitude Test 26-Item Version (the EAT-26) was used. The EAT-26 is a shortened version of the Eating Attitude Test (the EAT) developed by Gerner et al. (1982) for evaluating symptoms of ED. It is used worldwide not only for screening ED but also for measuring the degree of eating disturbances. The frequency of each item is rated on a 6-point Likert scale : “always," "usually," "often," "sometimes," "rarely," and “never," with three, two, and one point given to "always," "usually," and "often," respectively, and zero points assigned to the lower frequencies. The total score is calculated.

The cut-off point for Japanese has been reported as 15 points (Nakai, 2003), but no consensus has been reached. The score of 20 points suggested by Gerner et al. (1982) was adopted in this study, because its validity is recognized worldwide.

We extracted and used 26 items from the Japanese version of the EAT (Niisato et al., 1986).

2) Eating disturbance onset prediction test

Based on analyses of Preliminary Survey 1 and Preliminary Survey 2, the eating disturbance onset prediction test was developed.

(1) Preliminary Survey 1

We hypothesized eight concepts as essential risk factors through a review of longitudinal studies 
(Attie and Brooks-Gunn, 1989 ; Byely et al., 2000 ; Patton et al., 1999 ; Shisslak et al., 1999 ; Stice, 2002 ; Wichstrøm, 2000), and determined 82 items appropriate for non-clinical students from existing self-report questionnaires assessing these concepts, and modified them. These set of items and the EAT-26 were conducted with 328 female nursing college students in June and July, 2005.

Subjects were categorized into the eating disturbance group if their EAT-26 score was $\geq 20$, and into the normal eating group if their score was $<$ 20. The independent t-test was performed between the two groups. Factor analysis was performed on five concepts constructed by multiple items and factor loadings lower than 0.50 items were eliminated. Consequently, a total of 46 items remained, and 14 concepts including sub-concepts were found. Chronbach's coefficient alphas of 10 concepts constructed by multiple items including sub-concepts ranged from 0.747 to 0.905 .

A new concept, designated as "experience of teasing about body weight and shape," consisted of two sub-concepts, and items measuring this concept were added through additional literature review (Haines et al., 2006 ; Jackson et al., 2002 ; Keery et al., 2005). Eventually, nine concepts and 48 items measuring these concepts were used in the design of a questionnaire for the Preliminary Survey 2 .

(2) Preliminary Survey 2

Preliminary Survey 2 was conducted with 175 junior and 810 senior high school girls in October and November, 2005. Factor analysis was performed on the concepts constructed by multiple items, and factor loadings lower than 0.40 items were eliminated. Subjects were classified as described in Preliminary Survey 1 , and the independent $\mathrm{t}$-test was performed between the two groups. All items reached the 0.05 level of significance, except for one item belonging to the concept “perfectionism" ( $\mathrm{p}$ value $=0.131)$.

Finally, the following nine concepts were selected : (1) frequency of dieting, (2) thin-ideal internalization, (3) body dissatisfaction, (4) experience of teasing about body weight and shape, (5) perceived pressure to be thin, (6) self-esteem, (7) perfectionism, (8) life stressor, and (9) depression. As a result of factor analysis, two sub-concepts each were established in (2) thin-ideal internalization : (2) -1 modeling to be slim and (2)-2 awareness of social merit of being slim, (3) body dissatisfaction: (3)-1 general body dissatisfaction and (3) -2 dysfunction of social life attributed to body dissatisfaction, (4) experience of teasing about body weight and shape : (4)-1 impact of mild teasing and (4) -2 impact of severe teasing, (5) perceived pressure to be thin : (5)-1 impact of nonverbal pressure and (5) -2 impact of verbal pressure, and (6) self-esteem : (6)-1 global self-esteem and (6) -2 tendency of having self-esteem based on evaluation by others, and 3 sub-concepts in (7) perfectionism : (7)-1 anxiety about perfect accomplishment, (7) -2 concern over mistakes, and (7)3 a self-imposed high standard (Table 1 ).

A set of 38 items measuring above 16 concepts were termed the onset prediction test. Chronbach's coefficient alphas of 10 concepts constructed by multiple items ranged from 0.614 to 0.850 . Content validity was checked by a pediatric psychiatrist and a psychiatrist engaged in supervising school counseling.

All items were rated on a 4 -point Likert scale, and 1-4 points were given.

\section{Procedure}

The survey was conducted in April 2006 (T1) and about 10 months later in February 2007 (T2). At both times, the onset prediction test and the EAT-26 were distributed in the school, and the students were instructed by class or course teach- 
Table 1 The structure of the eating disturbance onset prediction test

\begin{tabular}{|c|c|c|}
\hline Concepts & Sub-concepts & The number of items \\
\hline (1) Frequency of dieting & & 1 \\
\hline \multirow[t]{2}{*}{ (2) thin-ideal internalization } & (2) -1 modeling to be slim $(\alpha=0.823)$ & 3 \\
\hline & (2) -2 awareness of social merit of being slim $(\alpha=0.768)$ & 3 \\
\hline \multirow[t]{2}{*}{ (3) body dissatisfaction } & (3) -1 general body dissatisfaction $(\alpha=0.815)$ & 3 \\
\hline & $\begin{array}{l}\text { (3) }-2 \text { dysfunction of social life attributed to body dissatisfaction } \\
(\alpha=0.788)\end{array}$ & 3 \\
\hline \multirow{2}{*}{$\begin{array}{l}\text { (4) experience of teasing about } \\
\text { body weight and shape }\end{array}$} & (4) -1 impact of mild teasing & 1 \\
\hline & (4) -2 impact of severe teasing & 1 \\
\hline \multirow{2}{*}{$\begin{array}{l}\text { (5) perceived pressure to be } \\
\text { thin }\end{array}$} & (5) -1 impact of nonverbal pressure & 1 \\
\hline & (5) -2 impact of verbal pressure & 1 \\
\hline \multirow[t]{2}{*}{ (6) self-esteem } & (6) -1 global self-esteem $(\alpha=0.636)$ & 3 \\
\hline & $\begin{array}{l}\text { (6) -2 tendency of having self-esteem based on evaluation by others } \\
(\alpha=0.850)\end{array}$ & 3 \\
\hline \multirow[t]{3}{*}{ (7) perfectionism } & (7) -1 anxiety about perfect accomplishment $(\alpha=0.617)$ & 4 \\
\hline & (7) -2 concern over mistakes $(\alpha=0.735)$ & 3 \\
\hline & (7) -3 a self-imposed high standard $(\alpha=0.614)$ & 3 \\
\hline (8) life stressor & & 1 \\
\hline \multirow[t]{2}{*}{ (9) depression $(\alpha=0.789)$} & & 4 \\
\hline & & total \\
\hline
\end{tabular}

ers following an instruction manual prepared by the researchers.

The students completed the onset prediction test and the EAT -26 at T1, and only the EAT -26 at T2. Of 1278 students, 1061 students who answered all items (effective answer rate : 83.0\%) were included in the analysis.

Students with an EAT-26 score of $\geq 20$ points were classified into the eating disturbance group, and those with a score of $<20$ points were classified into the normal eating behavior group. Based on the findings for the eating behavior groups at $\mathrm{T} 1$ and $\mathrm{T} 2$, the students were divided into four groups : onset-free group (eating behavior normal at $\mathrm{T} 1$ and $\mathrm{T} 2$ ), recovered group (eating disturbance group at $\mathrm{T} 1$, normal group at $\mathrm{T} 2$ ), onset group (normal group at $\mathrm{T} 1$, eating disturbance group at T2), and persistent group (eating disturbance group at $\mathrm{T} 1$ and $\mathrm{T} 2$ ).

For predictive discrimination of the onset group, a two-step discrimination protocol was employed In Step 1, EAT-26 items useful for the discrimination of the eating disturbance group already existing at $\mathrm{T} 1$ were investigated, and the normal group was extracted by excluding the eating disturbance group using the selected items. In Step 2, the normal group extracted in Step 1 was divided into the onset and onset-free groups using the selected onset prediction test items and items selected from the EAT-26 items employed in Step 1.

Approval for this study was obtained from the Ethics Committee of Osaka University School of Medicine. The students were instructed orally or in writing that answering the questionnaires was optional, and that school personnel and parents would never see the submitted questionnaires. The completed questionnaires were sealed in envelopes distributed with the questionnaires. Cooperating students filled in and submitted the questionnaires, and those who chose not to participate sub- 
mitted the questionnaires unanswered.

\section{Statistical Analysis}

Statistical analysis was performed with the 2step discrimination protocol using SPSS Version $13.0 \mathrm{~J}$ for Windows.

1) Step 1

Using the EAT-26 items at T1, canonical discriminant analysis was performed by the stepwise method. Thus, useful items for the discrimination of the eating disturbance group (recovered and persistent groups) and normal eating behavior group (the onset-free and onset groups) at $\mathrm{T} 1$ were investigated. Wilks' $\Lambda$ statistics were employed for the selection criteria, and a $\mathrm{p}^{-}$value $<0.05$ for canonical correlation was regarded as significant. The correct discrimination rate using the selected items (discrimination sensitivity and specificity) was analyzed. In addition, positive predictive values were calculated by the equation: (number of true positives/ (number of true positives + false positives $) \times 100$ ), and negative predictive values were calculated by the equation: (number of true negatives/ (number of true negatives + false negatives) $\times 100$ ) .

\section{2) Step 2}

The normal eating behavior group at $\mathrm{T} 1$ was subjected to canonical discriminant analysis by the stepwise method using the measurement items of the onset prediction test and the EAT-26 items extracted in Step 1. Consequently, useful items for the discrimination of the onset and onset-free groups were investigated. The selection criteria, correct discrimination rate analysis, and calculation of positive and positive predictive value were the same as in Step 1.

3) Power and meaning of the selected items

To investigate the onset risk of the selected items, the odds ratio and confidential interval were calculated with a level of significance of 0.05 . Fac- tor analysis of the selected items was performed by promax rotation.

\section{Results}

\section{Rates of the eating behavior groups}

At T1, 66 (6.2\%) and 995 (93.8\%) students were classified into the eating disturbance and normal eating behavior groups, respectively, and at T2, 79 (7.4\%) and $982(92.6 \%)$ students were classified into the eating disturbance and normal eating behavior groups, respectively. Based on the groups at $\mathrm{T} 1$ and $\mathrm{T} 2$, of the 1061 students, 950 (89.5\%), $32(3.0 \%), 34(3.2 \%)$, and $45(4.2 \%)$ students were classified into the onset-free, recovered, persistent, and onset groups, respectively (Table 2). Two students were previously treated for eating problems, but no subject was under treatment at the time of the surveys.

\section{Step 1 : Discrimination of the eating dis- turbance and normal eating behavior groups at $\mathbf{T} 1$}

The EAT-26 items useful for discrimination of the eating disturbance and normal eating behavior groups at T1 were investigated. Eleven items (EAT23, 21, 7, 18, 10, 12, 13, 17, 3, 25, and 26) were selected (Table 3 ). The correct discrimination rate using these 11 items was obtained as 95.5\% (discrimination sensitivity) in the eating disturbance group and as $95.9 \%$ (discrimination specificity) in the normal eating behavior group, and the overall false discrimination rate was $4.1 \%$ (44) 1061). The positive predictive value was $60.6 \%$, and the negative predictive value was $99.7 \%$ (Table 4).

\section{Step $2:$ Predictive discrimination of the onset and onset-free groups}

The item selection was repeated, and the following three discrimination patterns were obtained 
Table 2 Rates of 4 eating behavior groups

\begin{tabular}{|c|c|c|c|c|}
\hline & & \multicolumn{2}{|c|}{ Time 2} & \multirow[b]{2}{*}{ Total (\%) } \\
\hline & & $\begin{array}{l}\text { Eating disturbance } \\
\text { group }(\%)\end{array}$ & $\begin{array}{l}\text { Normal eating } \\
\text { behavior group }(\%)\end{array}$ & \\
\hline \multirow[t]{3}{*}{ Time 1} & $\begin{array}{l}\text { Eating disturbance } \\
\text { group }(\%)\end{array}$ & $\begin{array}{l}\text { Persistent group } \\
\quad 34(3.2)\end{array}$ & $\begin{array}{l}\text { Recovered group } \\
\quad 32(3.0)\end{array}$ & $66(6.2)$ \\
\hline & $\begin{array}{l}\text { Normal eating } \\
\text { behavior group }(\%)\end{array}$ & $\begin{array}{l}\text { Onset group } \\
45(4.2)\end{array}$ & $\begin{array}{l}\text { Onset-free group } \\
950\end{array}$ & $995(93.8)$ \\
\hline & Total (\%) & $79(7.4)$ & $982(92.6)$ & $1061(100.0)$ \\
\hline
\end{tabular}

Table 3 Standardization canonical discriminant function coefficient of the 11 items selected from the EAT-26 for Step 1

\begin{tabular}{lc}
\hline \multicolumn{1}{c}{ Selected Items } & Discriminant coefficient \\
\hline EAT23 : Engage in dieting behavior. & 0.226 \\
EAT21 : Give too much time and thought to food. & 0.303 \\
EAT7 : Particularly avoid food with a high carbohydrate content. & 0.291 \\
EAT18 : Feel that food controls my life. & 0.278 \\
EAT10 : Feel extremely guilty after eating. & 0.235 \\
EAT12 : Think about burning up calories when I exercise. & 0.210 \\
EAT13 : Other people think I'm too thin. & 0.207 \\
EAT17 : Eat diet foods. & 0.214 \\
EAT3 : Find myself preoccupied with food. & 0.249 \\
EAT25 : Enjoy trying new rich foods. & ${ }^{+}$ \\
EAT26 : Have the impulse to vomit after meals. & 0.231 \\
\hline
\end{tabular}

Items were arranged in rank order of selected by stepwise method.

${ }^{\dagger}$ : Reverse item. The reversed score was used for analysis.

Table 4 The result of discriminant analysis-Discrimination using the 11items selected from the EAT-26 for Step 1

\begin{tabular}{cccrr}
\hline & & \multicolumn{2}{c}{ Predicted group by discriminant analysis } & \\
\cline { 3 - 4 } & & $\begin{array}{c}\text { Eating disturbance } \\
\text { group }(\%)\end{array}$ & $\begin{array}{c}\text { Normal eating behavior } \\
\text { Troup }(\%)\end{array}$ & Total $(\%)$ \\
\hline \multirow{2}{*}{ The actual group } & Eating disturbance group $(\%)$ & $63(95.5)$ & $3(4.5)$ & $66(100.0)$ \\
& Normal eating behavior group $(\%)$ & $41(4.1)$ & $954(95.9)$ & $995(100.0)$ \\
\hline Total $(\%)$ & $104(9.8)$ & $957(90.2)$ & $1061(100.0)$ \\
\hline
\end{tabular}

The overall correct discrimination rate : $(63+954) / 1061 \times 100 \fallingdotseq 95.9 \%$

Positive predictive value : $63 /(63+41) \times 100 \fallingdotseq 60.6 \%$

Negative predictive value : $954 /(3+954) \times 100 \fallingdotseq 99.7 \%$

(Table 5).

1) Discrimination Pattern 1

Measurement items of the onset prediction test useful for discrimination of the onset and onsetfree groups were investigated, and the following four items were selected : (2)-2-(2) "To succeed in today's society, it is important for people to work hard on the body shape, avoiding getting fat," (3) -2-(2) "Thinking about own body shape interferes with your ability to concentrate on listening 
Jpn J Health \& Human Ecology

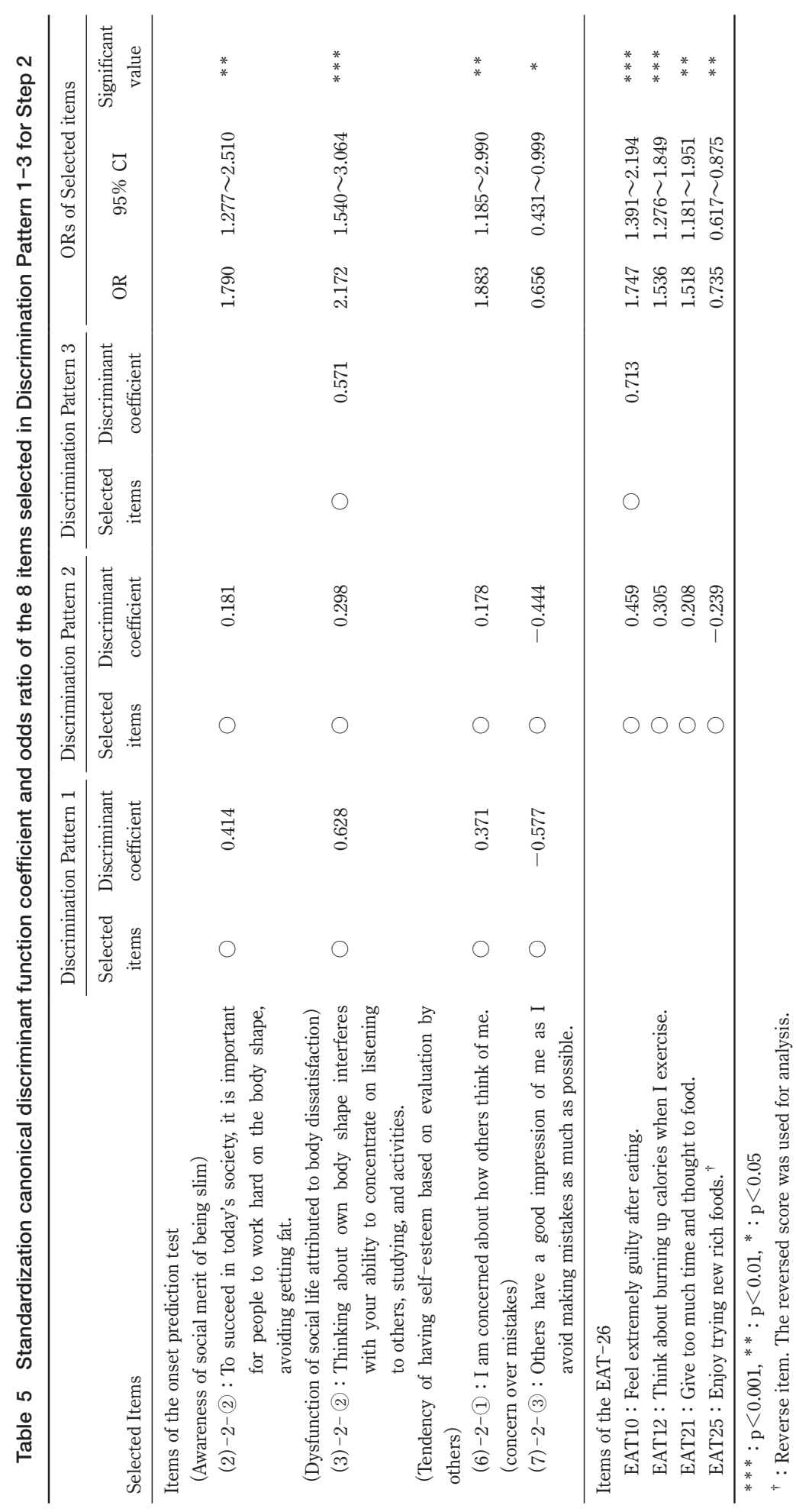


Table 6 The result of discriminant analysis-Discrimination using Discrimination Pattern 1

\begin{tabular}{ccrrrr}
\hline & & \multicolumn{3}{c}{$\begin{array}{c}\text { Predicted group by } \\
\text { discriminant analysis }\end{array}$} & \\
\cline { 3 - 4 } & & \multicolumn{2}{c}{$\begin{array}{c}\text { Onset } \\
\text { group (\%) }\end{array}$} & $\begin{array}{c}\text { Onset-free } \\
\text { group (\%) }\end{array}$ & \\
\hline \multirow{2}{*}{ The actual group } & Onset group (\%) & $33(73.3)$ & $12(26.7)$ & $45(100.0)$ \\
& Onset-free group (\%) & $265(27.9)$ & $685(72.1)$ & $950(100.0)$ \\
\hline Total (\%) & $298(29.9)$ & $697(70.1)$ & $995(100.0)$ \\
\hline
\end{tabular}

The overall correct discrimination rate : $(33+685) / 995 \times 100 \fallingdotseq 72.2 \%$

Positive predictive value : $33 /(33+265) \times 100 \fallingdotseq 11.0 \%$

Negative predictive value : $685 /(12+685) \times 100 \fallingdotseq 98.3 \%$

to others, studying, and activities," (6) -2-1 "I am concerned about how others think of me," and (7) 2-(3) "Others have a good impression of me as I avoid making mistakes as much as possible." The correct discrimination rate using these four items was obtained as $73.3 \%$ (predictive discrimination sensitivity) in the onset group and as $72.1 \%$ (predictive discrimination specificity) in the onset-free group, and the overall false discrimination rate was $27.8 \%$. Of the 45 students in the onset group, 12 students included in the onset-free group were false negative, accounting for $1.2 \%$ of all the students. The positive predictive value was $11.0 \%$, and the negative predictive value was $98.3 \%$ (Table 6).

\section{2) Discrimination Pattern 2}

Using the 11 EAT-26 items selected in Step 1, items useful for discrimination of the onset and onset-free groups were investigated, and four items (EAT10, 12, 25, and 21) were selected. Using eight items combining the four items selected in Discrimination Pattern 1, the correct discrimination rates were obtained as $68.9 \%$ (predictive discrimination sensitivity) in the onset group and $79.3 \%$ (predictive discrimination specificity) in the onset-free group. The overall false discrimination rate was $21.2 \%$. However, 14 students were false negative, accounting for $1.4 \%$ of all the students.
The positive predictive value was $13.6 \%$, and the negative predictive value was $98.1 \%$ (Table 7 ).

\section{3) Discrimination Pattern 3}

When discrimination was performed using two items, EAT10 and (3)-2-(2), the correct discrimination rate was obtained as $83.8 \%$ (predictive discrimination specificity) in the onset-free group, and the overall false discrimination rate was $18.0 \%$. However, the correct discrimination rate in the onset group was as $44.4 \%$ (predictive discrimination sensitivity), and 25 students were false negative, accounting for $2.5 \%$ of all the students. The positive and negative predictive values were $11.5 \%$ and $97.0 \%$, respectively (Table 8 ).

\section{Power of the items useful for discrimina- tion of the onset group and their interre- lationships}

Among the odds ratios of the eight items selected in the Discrimination Patterns $1-3$, two items were significantly directed toward risk reduction, and the odds ratios were 0.656 and 0.735 . Six items were significantly directed toward a risk increase, and the odds ratios ranged from 1.518 to 2.172 (Table 5).

On factor analysis, two factor structures were detected, and the following three items were constructed in the same factor : (2)-2-(2), (6)-2-(1, 
Table 7 The result of discriminant analysis-Discrimination using Discrimination Pattern 2

\begin{tabular}{|c|c|c|c|c|}
\hline & & \multicolumn{2}{|c|}{$\begin{array}{l}\text { Predicted group by } \\
\text { discriminant analysis }\end{array}$} & \multirow{2}{*}{ Total $(\%)$} \\
\hline & & $\begin{array}{c}\text { Onset } \\
\text { group }(\%)\end{array}$ & $\begin{array}{l}\text { Onset-free } \\
\text { group }(\%)\end{array}$ & \\
\hline \multirow[t]{3}{*}{ The actual group } & Onset group (\%) & $31 \quad(68.9)$ & $14(31.1)$ & $45(100.0)$ \\
\hline & Onset-free group $(\%)$ & $197(20.7)$ & $753(79.3)$ & $950 \quad(100.0)$ \\
\hline & Total (\%) & $228(22.9)$ & $767(77.1)$ & $995(100.0)$ \\
\hline
\end{tabular}

The overall correct discrimination rate : $(31+753) / 995 \times 100 \fallingdotseq 78.8 \%$

Positive predictive value : $31 /(31+197) \times 100 \fallingdotseq 13.6 \%$

Negative predictive value : $753 /(14+753) \times 100 \fallingdotseq 98.1 \%$

Table 8 The result of discriminant analysis-Discrimination using Discrimination Pattern 3

\begin{tabular}{|c|c|c|c|c|}
\hline & & \multicolumn{2}{|c|}{$\begin{array}{l}\text { Predicted group by } \\
\text { discriminant analysis }\end{array}$} & \multirow{2}{*}{ Total $(\%)$} \\
\hline & & $\begin{array}{c}\text { Onset } \\
\text { group (\%) }\end{array}$ & $\begin{array}{l}\text { Onset-free } \\
\text { group }(\%)\end{array}$ & \\
\hline \multirow[t]{3}{*}{ The actual group } & Onset group (\%) & $20(44.4)$ & $25(55.6)$ & $45(100.0)$ \\
\hline & Onset-free group $(\%)$ & $154(16.2)$ & $796(83.8)$ & $950(100.0)$ \\
\hline & Total (\%) & $174(17.5)$ & $821 \quad(82.5)$ & $995(100.0)$ \\
\hline
\end{tabular}

The overall correct discrimination rate : $(20+796) / 995 \times 100 \fallingdotseq 82.0 \%$

Positive predictive value : $20 /(20+154) \times 100 \fallingdotseq 11.5 \%$

Negative predictive value : $796 /(25+796) \times 100 \fallingdotseq 97.0 \%$

and $(7)-2-3$.

\section{Discussion}

\section{Rates of the eating behavior groups}

In this longitudinal study, the eating behavior of adolescent girls was classified. Several studies have transversely evaluated the eating behavior of adolescent girls, but there have been very few studies in which subjects were classified based on time-course changes in eating behavior. Deshimaru et al. (2002) performed a longitudinal study, in which the eating behavior of high school girls was classified by setting the cut off to 20 points on the EAT-26, similar to our study. They found that the ratios of the onset-free, recovered, persistent, and onset groups were $90.23 \%, 2.82 \%, 3.01 \%$, and $3.95 \%$, respectively. They surveyed changes after one year in September in more than one grade of high school girls. We surveyed only firstyear students in April, considering that screening aimed at preventive measures can be performed early after entering high school. To assess the effectiveness of this strategy, changes after 10 months were investigated. Thus, direct comparison of the present study with Deshimaru et al. (2002) is not possible, although it may be noteworthy that the ratios of the eating behavior groups in both studies were similar. This suggests that the tendency of eating behavior of high school girls was consistent with the ratios in our study to some extent. 


\section{Feasibility of screening the eating distur- bances onset group}

With a high incidence of ED, the age has been reported to have a bimodal distribution (14 and 18 years of age) (Halmi et al., 1979), or 17 years of age (Hindler et al., 1994; Nakai, 2000), and the prognosis becomes poorer as the onset age increases (Nakai et al., 2002). Thus, avoidance of the onset at 17-18 years of age should be set as a target of prevention. It is very important to take preventive measures in stages earlier than these ages through preventive education, etc.

As described previously, selected prevention programs have a higher preventive effect than universal prevention programs (Stice and Shaw, 2004). In addition, negative influences of the attendance of students at low risk on those at high risk at places of preventive education were reported, and preventive education on students at low risk was rather than harmful (Abascal et al., 2004). Based on the effects of preventive education, such education performed earlier than 17-18 years of age should be limited to students predicted to be at a high risk of developing eating disturbances, and extraction of such students should be investigated.

Screening using the EAT-26 items has been proposed because the score of the EAT-26 indicates the degree of eating disturbances, and several screening methods using some items have been investigated (Hayashi et al., 2004 ; Hisamatsu et al., 2000 ; Nagata et al., 1989). However, such screenings were aimed at the detection of students already belonging to the eating disturbance group, but not at the extraction of students predicted to develop such eating disturbances, which was the purpose of our study. Since the EAT-26 has high reliability and validity for measurement of the degree of current eating disturbances (Garfinkel and Newman, 2001), we attempted to design a discrimination protocol employing the EAT-26 items for discriminating the eating disturbance onset group. The reason for the employing was items related to eating behavior and dietary cognition were not included in the onset prediction test, except for (1) "frequency of dieting."

When the 11 EAT-26 items selected for Step 1 were used, the discrimination sensitivity and specificity were found to be good. Therefore, it is clear that discrimination of the eating disturbance and normal groups is possible using the 11 EAT-26 items.

Using the four onset prediction test items selected for Step 2 in Discrimination Pattern 1, $73.3 \%$ of onset group students could be discriminated, although items measuring 10 months earlier eating behavior and dietary cognition were absent. This finding supports previous studies reporting that not all students with aberrations in eating behavior and dietary cognition develope $\mathrm{ED}$, with predisposing factors such as individual personality factors likely producing the differences (Schleimer, 1983 ; Stice, 2002 ; Striegel-Moore et al., 1989 ; The McKnight Investigators, 2003).

It is beneficial that three of four students with normal eating behavior who will develop eating disturbances in the future can be identified using only four items, however, it cannot be ignored that false positives accounted for $26.6 \%$ of all the students. In Discrimination Pattern 2, items related to eating behavior and dietary cognition were added to the four items to reduce the false-positive rate. Since it is important to discriminate using a minimum number of measurement items in the primary care setting, useful items were investigated in the 11 EAT-26 items selected for Step 1. In Discrimination Pattern 2, the correct discrimination rates were improved, and the rate of false positive students was reduced. However, the correct discrimination rate of the onset group was reduced, and false negative students were elevated. 
Discrimination Pattern 3 led to good correct discrimination rates using only two measurement items. However, the rate of false negative students was the worst of the three discrimination patterns, showing it inappropriate for primary screening.

Out of the onset prediction test, no items of (3)1 "general body dissatisfaction," (2) -1 “modeling to be slim," or (1) "frequency of dieting" were selected. This suggests that students' dissatisfaction with their own body, preference of a slim person as a role model, and dieting is applicable in the case of not only adolescent girls developing eating disturbances but also those without such disturbances. Similar findings have been reported by others (Nagata et al., 1991 ; Patton, 1988 ; Whitehouse et al., 1988). Nagata et al. (1989) performed discriminant analysis of individuals diagnosed with anorexia nervosa or bulimia nervosa, and healthy subjects using the EAT, and selected items not belonging to the subscale "dieting." They showed that desire to be slim and a dislike of being fat are widely present throughout the society, and warned of the link between dieting due to a dislike of being fat and ED. Therefore, it is appropriate that these items are not selected in this study. Some preliminary studies have suggested that Japanese girls show a higher level of body dissatisfaction than European and American girls (Nakai, 1997 ; Kashima et al., 2003). Therefore, for the prediction of ED and eating disturbances in Japanese girls, items related to (3) -1 should be excluded.

However, (2) 2 "awareness of social merit of being slim" and (3) -2 "dysfunction of social life attributed to body dissatisfaction" were selected as useful items for the prediction of the onset group. The odds ratio of (3)-2-(2)was 2.172, and the onset risk increased more than two times as the degree increased by one rank. Although these appeared to be similar to (2) -1 "modeling to be slim" and (3) -1 "general body dissatisfaction," their implications were completely different. In other words, (2) -1 and (3) - 1 represent mental images of individuals, whereas (2) -2 and (3) -2 are communicational phenomena or attitudes of individuals toward the society based on these mental images. In the majority of studies designed to investigate the relationship between desire to be slim or body dissatisfaction and ED, desire to be slim meant "modeling to be slim," and body dissatisfaction meant "general body dissatisfaction." However, our study clarified that desire to be slim should include the aspect of awareness of the social merit of being slim, and body dissatisfaction should include the aspect of dysfunction of social life attributed to body dissatisfaction. Thereby, this leads us to obtain qualitative suggestions about the development process of $\mathrm{ED}$ and disturbances.

The selection of (6)-2-1)also indicates that the communicational attitude of individuals toward society is different between the onset and onset-free groups.

Concerning the selection of (7)-2-(3) "concern over mistakes," an unexpected finding was the increase in the risk of developing eating disturbances as the score decreased. We included (7) -2(3) in the onset prediction test items as a sub-concept of "perfectionism," assuming that the predisposing risk of eating disturbances increases as the degree of perfectionism increases, but an opposite finding was obtained. This indicates that the group developing eating disturbances has a tendency to consider that making mistakes is not related to the impression they give of themselves. On the factor analysis, (2)-2-(2), (6)-2-1), and (7)-2-(3)were classified as the same factor, which suggests a possibility that the onset group thinks that "whether others have a good impression of me depends on being thin and appearance." The factor made up of these three items can be named "the communica- 
tional attitude of individuals toward society," and is worth paying attention to as a concept characterizing the onset group.

Out of the 11 items selected from the EAT-26 for Step 1, EAT10, 12, 21, and 25 were selected in Discrimination Patterns 2 and 3. In previous studies on screening using some of the EAT-26 items, EAT21 was selected by Nagata et al. (1989), EAT10 and 21 by Hisamatsu et al. (2000), and EAT12 and 21 by Hayashi et al. (2004). The four EAT-26 items partially overlapped with these previous studies, indicating that focusing on specific eating behavior and dietary cognition of patients with ED and related symptoms is beneficial, and that such behaviors and cognitions are common in $\mathrm{ED}$ and eating disturbances. These findings $\mathrm{re}^{-}$ confirm that ED and eating disturbances are continuous concepts.

EAT25 was a reverse item selected by our study, which was not selected in the previous studies cited above. The odds ratio of this item was 0.735 , and the risk of developing eating disturbances increased as the reversed score decreased, indicating that the onset group is more interested in eating than the onset-free group, and they behave like this in their daily lives. Strong interest in eating, existing as a preparatory condition, may be an important factor in the development process of eating disturbances ; it may increase conflict in dieting and is likely to induce inappropriate compensatory behavior such as binge eating and purging. This may reflect the current era of gluttony. Detailed clarification of the contemporary background of the development process of eating disturbances is necessary.

\section{Utilization for preventive measures}

This study was performed to extract screening items, which elevate the overall correct discrimination rate while keeping both the predictive dis- crimination sensitivity and specificity at high levels. For the detection of students needed to be delivered preventive intervention, the false-negative rate should be low so that the purpose of screening is achieved even if the false-positive rate is somewhat high. On the other hand, when the disease prevalence is low, it is difficult to increase the positive predictive value, and elevation of the negative predictive value by increasing the specificity is effective for increasing the predictive value of screening in a population with a low prevalence (Gordis, 2002). In the screening using Discrimination Pattern 1, although the positive predictive value was low, the negative predictive value was highest among the three discrimination patterns and the false negative was the lowest. Therefore, for screening to identify students needed to be delivered preventive measures, it may be appropriate to apply Discrimination Pattern 1 and extract the group predicted to be the onset group as required for preventive intervention.

In brief, the following protocol was finally discovered. Eating disturbance group students at T1 are extracted using the 11 EAT-26 items in Step 1, for whom secondary prevention activity is assigned, and the onset group students are extracted from the remaining normal eating behavior group using the 4 onset prediction test items in step 2, for whom primary prevention activity is assigned (Fig. 1). This protocol would exclude more students from primary preventive measures than universal prevention programs, for instance more than $70 \%$ of the students in this study. This discrimination protocol allows selected prevention programs, which may lead to the efficient practice of effective preventive education well suited for the risk.

However, we must note that false negative students accounting for $1.2 \%$ of all the students were excluded from preventive education. Further inves- 


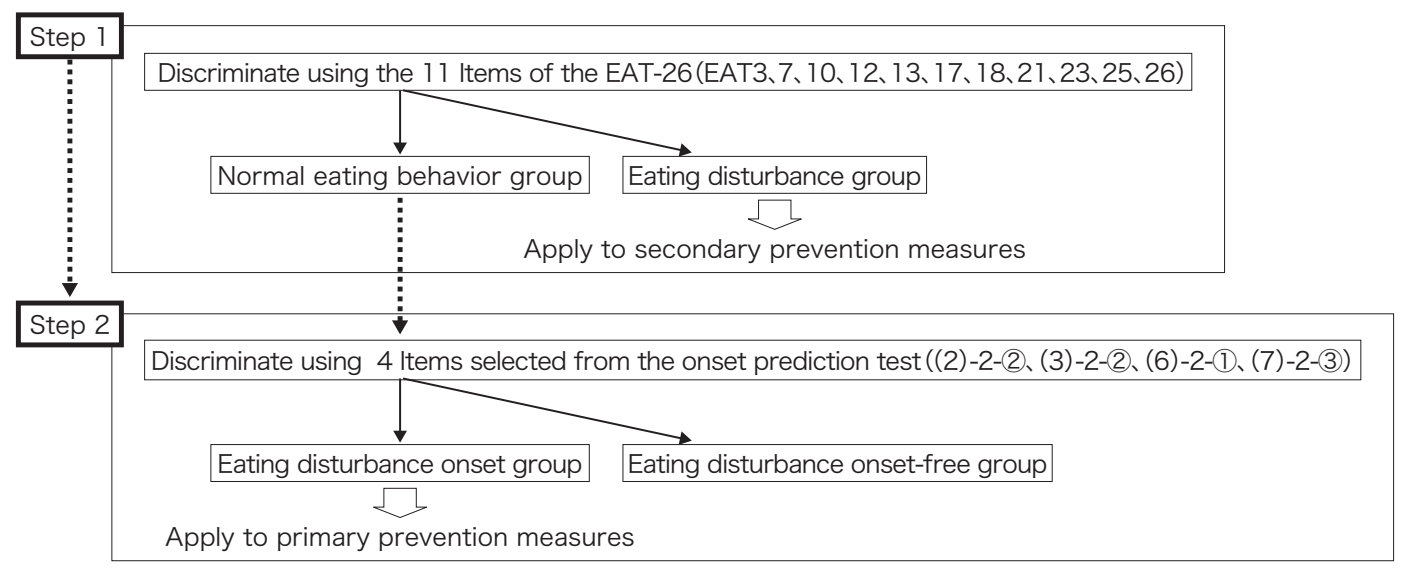

Figure 1 Discrimination protocol for predicting onset of eating disturbances

tigation of the discrimination method to reduce the false negative rate is necessary to apply this protocol to screening aimed at preventive measures.

\section{Limitation}

In this study, the eating disturbance group targeted for screening is a group with severe symptoms almost similar to those of ED, and students in whom the EAT -26 score was $<20$ points at $\mathrm{T} 1$ rose to $\geq 20$ points at $\mathrm{T} 2$ were extracted. However, when eating disturbances are considered to be present in a continuous course from normal eating to full-syndrome ED, the severity of eating disturbances varies. Thus, further considerations of the severity for the screening are needed. We deal with one screening criteria, and attempted to predict the onset in the extracted group. The result may vary depending on the criteria, but the findings regarding the discrimination method applied to one of several conceivable screening criteria represent a useful step in the ongoing effort to seek a better screening protocol.

A limitation of our study is that the results were obtained from only seven schools in large urban areas. No regional differences of the eating disorder incidence at present were reported, although the incidence of $\mathrm{ED}$ has been reported to be higher in urban areas (Rathner and Messner, 1993). However, it is still needed to select subjects from various types of school in different areas, and accumulate data to increase the reliability of surveys.

\section{Conclusion}

The discrimination protocol using the 11 EAT26 items and the four onset prediction test items is useful for predicting the onset of eating disturbances with time in first-year female high school students. To apply this protocol to screening aimed at preventive measures, reduction of the falsenegative rate by modification of measurement items may be needed.

\section{Acknowledgements}

We are grateful to the respondents who took the time to participate in our survey. This article is based on the first author's doctoral dissertation. This research was supported in part by ResearchAid, Meiji Yasuda Mental Health Foundation and the Yamaji Fumiko Nursing Research Fund, Japan. 


\section{References}

Abascal L, Brown JB, Winzelberg AJ, et al. (2004) : Combining universal and targeted prevention for school-based eating disorder programs, Int J Eat Disord, 35, 1-9

Attie I and Brooks-Gunn J (1989) : Development of eating problems in adolescent girls : a longitudinal study, Dev Psychol, 25, 70-79

Bunnell DW and Shenker IR (1990) : Subclinical versus formal eating disorders : differentiating psychological features, Int J Eat Disord, 9, 357-362

Byely L, Archibald AB, Graber J, et al. (2000) : A prospective study of familial and social influences on girls' body image and dieting, Int J Eat Disord, 28, 155-164

Crow SJ, Stewart AW, Halmi K, et al. (2002) : Full syndromal versus subthreshold anorexia nervosa, blimia nervosa, and binge eating disorder: a multicenter study, Int J Eat Disord, 32, 309-318

Deshimaru M, Yamaguchi H, Harai H, et al. (2002) : Kikuiketoshi no koukou deno setsushokushougai no jyoukyou, Kouseiroudoushou seishinshinkeishitsukan kenkyuitakuhi niyoru 13nendo kenkyuhoukokusho setsushokushougai no chiryoujyoukyou yogotou ni kansuru chousakenkyu, 91-102, (Tokyo)

Garfinkel PE and Newman A (2001) : The eating attitudes test : twenty-five years later, Eat Weight Disord, 6, 1-24

Gerner DM, Olmstead MP and Bohr Y (1982) : The Eating Attitude Test:psychometric features and clinical correlates, Psychol Med, 12, 871-878

Gordis L (2002) : Epidemiology 2nd Edition, 69-74, WB Saunders Co (Philadelphia)

Haines J, Neumark-Sztainer D, Eisenberg ME, et al. (2006) : Weight teasing and disordered eating behaviors in adolescents : longitudinal findings from Project EAT (Eating Among Teens), Pediatrics, 117, e209-e215

Halmi KA, Casper RC, Eckert ED, et al. (1979) : Unique features associated with age of onset of anorexia nervosa, Psychiatry Res, 1, 209-215

Hayashi Y, Kosugi S, Shimazu A, et al. (2004) : Development of primary screening test for eating disorders in a health examination for students-Item selection and validity, Jpn J school Health, 46, 254-263

Hindler CG, Crisp AH and McGuigan S et al. (1994) : Anorexia nervosa; change over time in age onset, presentation and duration of illness, Psychol Med, 24, 719-729

Hisamatsu Y, Tsuboi K, Tsutsui S, et al. (2000) : A study on primary screening method for eating disorder in female university students, Jpn J Psychosom Med, 40, 325-331

Jackson TD, Grilo CM and Masheb RM (2002) : Teasing history and eating disorder features : an age-and body mass index-matched comparison of bulimia nervosa and binge-eating disorder, Compr Psychiatry, $43,108-113$

Johnson JB, Cohen P, Kasen S, et al. (2002) : Eating disorders during adolescence and the risk for physical and mental disorders during early adulthood, Arch Gen Psychiatry, 59, 545-552

Kashima A, Yamashita T, Okamoto A, et al. (2003) : Japanese version of the Body Attitude Test: Its reliability and validity, Psychiatry Clin Neurosci, 57, 511516

Keery H, Boutelle K, van den Berg P, et al. (2005) : The impact of appearance-related teasing by family members, J Adolesc Health, 37, 120-127

Kiriike N (2005) : Eating disorders, Seishinigaku, 47, 145-149

Mitrany E (1992) : Atypical eating disorders, J Adolesc Health, 13, 400-402

Nagai M, Aoki K, Masuda K, et al. (2005) : The primary prevention program for eating disorders among high school girls, Jpn J School Health, 47, 436-451

Nagata T, Kiriike N, Nakanishi S, et al. (1991) : Development and application of the new symptom rating scale for eating disorders (SRSED), Seishinkashindangaku, 2, 247-258

Nagata T, Kiriike N, Yoshino S, et al. (1989) : Reliability and validity of the Eating Attitudes Test in patients with eating disorders, Jpn J Clin Psychi, 18, $1279-1286$

Nakai Y (1997) : Eating Disorder Inventory scores in eating disorders, Seishinigaku, 39, 47-50

Nakai Y (2000) : Epidemiology of eating disorders, Psychosom Med, 4, 1-9

Nakai Y (2003) : Validity of Eating Attitudes Test, Clinical Psychi, 45, 161-165

Nakai Y, Hamagaki S, Ishizaka Y, et al. (2002) : Predictors of outcome in eating disorders, Clinical Psychi, 44, 1305-1309

Niisato S, Tamai H, Fujii S, et al. (1986) : A development of/and validity and reliability studies of the Japanese version of Eating Attitudes Test, Shinshinigaku, 26, 398-407

Patton GC (1988) : The spectrum of eating disorder in adolescents, J Psychosom Res, 32, 579-584

Patton GC, Selzer R, Coffey C, et al. (1999) : Onset of adolescent eating disorders : population based cohort 
study over 3 years, BMJ, 318, 765-768.

Rathner G and Messner K (1993) : Detection of eating disorders in small rural town; an epidemiological study, Psychol Med, 23, 175-184

Schleimer K (1983) : Dieting in teenage schoolgirls-A longitudinal prospective study, Acta Pediatr Scand, 312 (Suppl), 1-54

Shisslak CM, Renger R, Sharpe T, et al. (1999) : Development and evaluation of the McKnight Risk Factor Survey for assessing potential risk and protective factors for disordered eating in preadolescent and adolescent girls, Int J Eat Disord, 25, 195-214

Stice E (2002) : Risk and maintenance factors for eating pathology : a meta-analytic review, Psychol Bull, $128,825-848$

Stice E and Shaw H (2004) : Eating disorder prevention programs : a meta-analytic review, Psychol Bull, 130, 206-227

Striegel-Moore RH, Siberstein LR, French P, et al. (1989) : A prospective study of disorderd eating among college students, Int J Eat Disord, 8, 499-509

Tanaka T, Ishii K, Hirokane K, et al. (2004) : Early diagnosis for anorexia nervosa in school health practice, Keio Hoken Kenkyu, 22, 55-59

The Mcknight Investigators (2003) : Risk factors for the onset of eating disorders in adolescent girlsResults of the McKnight Longitudinal Risk Factor Study, Am J Psychiatry, 160, 248-254

Watanabe H and Tokumura M (2005) : Prevention and early intervention in school health practice, Guide for diagnosis and treatment of anorexia nervosa, 38-53, Bunkodo, (Tokyo)

Weber CC, Narring F and Michaud PA (2005) : Partial eating disorders among adolescents : A review, J Adolesc Health, 37, 417-427

Whitehouse AM, Freeman C and Annandale A (1988) : Body size estimation in anorexia nervosa, Br J Psychiatry Suppl, 152, 23-26

Wichstrøm L (2000) : Psychological and behavioral factors unpredictive of disordered eating : a prospective study of the general adolescent population in Norway, Int J Eat Disord, 28, 33-42

(Received 9.21.2007 ; Accepted 12.17.2007) 